\title{
A feasibility study of individual 3D-printed navigation template for the deep external fixator pin position on the iliac crest
}

Bin Liang ${ }^{1+}$, Qiang Chen ${ }^{2 \dagger}$, Shuai Liu ${ }^{3,4}$, Shuo Chen ${ }^{5}$, Qingqiang Yao ${ }^{1,3}$, Bo Wei ${ }^{1,3}$, Yan X X ${ }^{1,3}$, Cheng Tang ${ }^{1,3^{*}}$ and Liming Wang ${ }^{1,3^{*}}$

\begin{abstract}
Background: The aim of this study was to investigate the feasibility of an individual navigation template for the deep pin position on the iliac crest, based on digital design and 3D printing technology.

Methods: The preoperative CT images of 8 patients with pelvic fractures were collected. The data were reconstructed using a 3D imaging reconstruction workstation. An individual navigation template for the deep pin position on the iliac crest was designed on a virtual 3D model. The individual drill template and the solid pelvic model were produced using the 3D printing technology. The individual drill template was used for intraoperative deep pin position on the iliac crest after the preoperative simulation was completed.

Results: Thirty-two external fixator pins were inserted using the individual drill template. The average depth of pins was $84.82 \mathrm{~mm}$. The trajectories were appropriate based on the postoperative X-ray and CT scan. No significant difference in the entry point, convergence angle, and caudal angle of the pins were noted before and after the operation (all $P>0.05$ ). Finite element analysis indicated that the deep external fixator pin position could more reasonably distribute the stress in the cortical and spongy bones in the pelvis. All patients could perform partial weight-bearing activity 6 weeks postoperatively. No loosening and rupture of the pin, infection, and no damage of blood vessels and nervous tissue were found during the entire follow-up period.

Conclusions: The individual drill template technique is an improvement of the traditional technique, which could increase precision and the depth of pin position. In addition, good mechanical stability and low risk of pin-related complications occurred due to the individual drill template, which makes the external fixation technique a potential alternative.
\end{abstract}

Keywords: Individual, Template, Pelvis fracture, External fixators

\footnotetext{
*Correspondence: tangc233@njmu.edu.cn; limingwang99@yahoo.com

${ }^{\dagger}$ Bin Liang and Qiang Chen should be considered joint first author

'Department of Orthopaedic Surgery, Nanjing First Hospital, Nanjing Medical University, Nanjing 210006, China

Full list of author information is available at the end of the article
}

(c) The Author(s). 2020 Open Access This article is licensed under a Creative Commons Attribution 4.0 International License, which permits use, sharing, adaptation, distribution and reproduction in any medium or format, as long as you give appropriate credit to the original author(s) and the source, provide a link to the Creative Commons licence, and indicate if changes were made. The images or other third party material in this article are included in the article's Creative Commons licence, unless indicated otherwise in a credit line to the material. If material is not included in the article's Creative Commons licence and your intended use is not permitted by statutory regulation or exceeds the permitted use, you will need to obtain permission directly from the copyright holder. To view a copy of this licence, visit http://creativecommons.org/licenses/by/4.0/ The Creative Commons Public Domain Dedication waiver (http://creativecommons.org/publicdomain/zero/1.0/) applies to the data made available in this article, unless otherwise stated in a credit line to the data. 


\section{Background}

Pelvic fractures are usually caused by high-energy trauma, such as traffic accident or falls from heights in young population, and with a consequent of associated injuries. In the elderly population with osteoporosis, fragility fractures can often be caused by low-energy trauma, such as a fall from standing or some minor trauma. The overall mortality rate worldwide varies from 6 to $35 \%[1,2]$. External fixation plays an important role in the reduction of pelvic volume, pain, and bleeding for the high-energy trauma and the fragility fracture. The traditional external fixation on the iliac crest is the commonly used surgical technique because it is minimally invasive and easy to perform [3]. However, it is difficult to control the direction of external fixator pins using the traditional surgical technique, which often results in the pin tip easily penetrating the cortex of the ilium during the surgery. In contrast with our experience using the percutaneous approach, correct pin placement using the anterosuperior approach is particularly difficult for inexperienced surgeons, Wailakul et al. reported that the incidence rate of incorrect pin placement up to $18 \%$ at the iliac crest for external fixation [4]. In addition, the incorrect pins are prone to infection and loosening, which greatly affects the mechanical stability of external fixation [5]. It is reported that the strong pin purchase can be achieved due to the high bone density in the supraacetabular region of human pelvis [6]. We wanted to investigate a simple modification of the traditional method to place pin from the iliac crest toward the supraacetabular region, thus to improve the mechanical stability of external fixation. To the best of our knowledge, there are no reported studies improving the current surgical technique until now [7].

Rapid prototyping (RP) is an emerging industrial technique. Its application combined with reverse engineering technology in the field of medicine has made precise and individualized treatment possible. The technique is especially important in case of minimally invasive surgeries. Preoperative design and surgical planning, along with the application of a 3D-printed navigation template, can effectively reduce errors associated with lack of experience and poor operative techniques. Currently, navigation templates are used to guide the surgical insertion of internal fixation screws and plates. However, its application in the deep pin positioning on the iliac crest has not been reported. The aim of this study was to use digital 3D reconstruction, reverse modeling technology, and $3 \mathrm{D}$ print to produce an individual navigation template. The template was designed to serve as a guide to increase the precision and depth of external fixator pin positioning on the iliac crest.

\section{Methods}

\section{General information}

This research was authorized by Nanjing First Hospital's ethics committee. Eight pelvic-fracture patients refused expectant treatment and selected minimally invasive surgery. They were given detailed information about the study and their situation and signed the informed consent forms. The 3D navigation template was used as part of the surgical procedure for all patients. The general clinical data of the patients are listed in Table 1.

\section{Preoperative measurement, design, and template preparation}

All experimental methods described in this study were approved by Nanjing First Hospital and performed in accordance with the relevant guidelines and regulations. A spiral CT scan of pelvis was obtained using the following specific scan parameters: $120 \mathrm{KV}, 120 \mathrm{mAs}$, pixel matrix $512 \times 512,1 \mathrm{~mm}$ thick slices, $0.5 \mathrm{~mm}$ interlamellar spacing. Original DICOM data were imported into Mimics software (Materialise Inc., Belgium) to produce a 3D reconstruction of pelvis. Optimal pin position in simulation module is facilitated by searching a position with adequate depth and avoiding bone penetration in the 3D reconstruction of pelvis. These data were imported into the 3D reconstruction program (STL format) in Geomagic Design Direct software to redefine the coordinate axis. Then, the trajectories of the external fixator pin were determined [8]. A navigation template with reverse modeling was designed, and the navigation template 3D model was completed with the insertion channel for the pin (Fig. 1). The template and pelvis model were produced by the fused deposition modeling (FDM) method of RP technology.

Following the preparation of the template and pelvis model, the simulated operation was performed. After matching navigation templates to the pelvic models, the external fixator pins were inserted through the insertion channel. The position and orientation of the pin were evaluated according to the preoperative plan. The templates were sterilized with low-temperature plasma before operation.

\section{Operation and postoperative treatment}

Using a cambered incision along the iliac crest, the soft tissue was carefully separated to reveal the base of the iliac crest and the inner plate of the ilium. The 3D navigation template was used to guide the insertion of the pin as described above. After all pins have been inserted, a low-profile and anterior frame was then constructed and angled caudally. The pin-bar clamps were attached to the pins, then short connecting rods were fastened to the pins. Long rods were attached to the short connecting rods, then connected by bar-bar clamps. Double 
Table 1 The baseline characteristics of patients

\begin{tabular}{|c|c|c|c|c|c|c|c|}
\hline No. & Age & Gender & BMI & Location of injury & Cause of injury & AO/OTA & Time from trauma to surgery \\
\hline 1 & 61 & Female & 23.5 & Both sides & Car accident & B1 & 6 days \\
\hline 2 & 65 & Female & 29.1 & Right side & Car accident & B1 & 6 days \\
\hline 3 & 29 & Male & 26.7 & Right side & Fall & $\mathrm{C} 1$ & 7 days \\
\hline 4 & 42 & Male & 25.8 & Left side & Car accident & B2 & 4 days \\
\hline 5 & 22 & Female & 28.4 & Left side & Car accident & B2 & 1 days \\
\hline 6 & 61 & Male & 26.3 & Left side & Car accident & B1 & 3 days \\
\hline 7 & 80 & Male & 31.2 & Both sides & Car accident & $\mathrm{C} 1$ & 3 days \\
\hline 8 & 58 & Male & 24.9 & Right side & Fall & $\mathrm{C} 1$ & 2 days \\
\hline
\end{tabular}

stacking the connecting rods were applied to increase frame stability.

According to the preoperative and postoperative 3D reconstructions, three parameters (entry point, convergence angle, and caudal angle) of all pins were measured and compared before and after the operation, respectively, to evaluate whether the trajectories were precise and consistent with the preoperative plans. The entry point refers to the distance from the entry point to the anterior superior iliac spine. The convergence angle refers to the axis of the pin to the sagittal plane of the pelvis. The caudal angle refers to the axis of the pin relative to the plane, which is the axis of the true conjugate (from sacral promontory to symphysis) vertical to the sagittal plane.

Postoperatively, the patients remained touch-down for 6 weeks, but there were no restrictions regarding hip motion. At 6 weeks, the patients were assessed for fracture consolidation according to regional symptoms and signs, as well as X-ray findings which included callus formation, fracture displacement and implant position. Then the patients were generally allowed to be partial weight bearing. After 12 weeks, when the fracture had mostly healed, the external fixator pins were removed and thereafter advanced to the full weight bearing.

\section{The finite element model and mechanical analysis}

To analyze the effect of different insertion depths of the external fixator pin on the stress distribution of pelvis, two insertion depths $30 \mathrm{~mm}$ and $70 \mathrm{~mm}$ were chosen for finite element analysis. The inserting direction of pins designed in finite element analysis was consistent with that in the surgery, as well as the distance between the entry points of the pelvis and the connectors which connected the pins and the frame. Based on the postoperative CT images (slice number: 489, slice thickness: 1 $\mathrm{mm}$ ) of a pelvis-fractured patient, the finite element model of the pelvis was reconstructed using the 3D

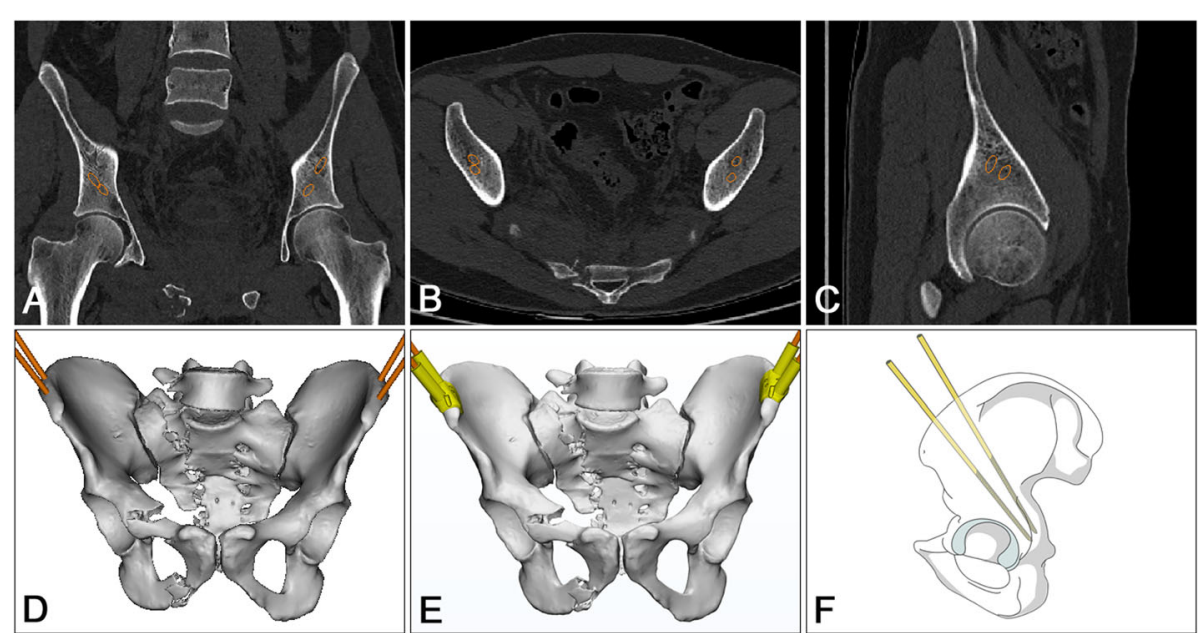

Fig. 1 Preoperative design and template preparation. a-d DICOM data were imported into Mimics software for 3D reconstruction, and external fixator pin insertion channels were simulated. e The navigation template was designed with reverse modeling. $\mathbf{f}$ Ideal position of external fixator pins 
medical image processing software Mimics (Materialise Inc., Belgium). It is worth mentioning that in the model reconstruction, we manually reunited the fractured parts to guarantee its mechanical stability, and then focused on the stress distribution caused by the pin insertion. Four identical external fixator pins made by stainless steel 305 with $5 \mathrm{~mm}$ in diameter and $124-175 \mathrm{~mm}$ in length were modeled using another commercial software Abaqus (Dassault Systèmes Simula, USA).

The pelvis was geometrically defined as a composite structure with a $3 \mathrm{~mm}$ thickened shell of cortical bone enclosing spongy bone [9]. Here, the sacrum was only considered to be consisted of cortical bone, as we mainly focused on the stress distribution of the fractured pelvis caused by different pin-inserting depths. All the materials were considered to be linear-elastic and isotropic, and their parameters were listed in Table 2. The shell element S3 was assigned to the cortical part in the pelvis, and the solid element C3D4 was assigned to the remaining structures including spongy bone in the ilium, sacrum, and the fixator pin. For the assembled model, the total element number in case of insertion $30 \mathrm{~mm}$ was 227,299 (C3D4: 178952, S3: 48347), and that in case of insertion $70 \mathrm{~mm}$ was 204, 071 (C3D4: 162862, S3: 41209). In the simulation, the contact of cortical bone and spongy bone in the ilium was set to be coupled, and that of the sacrum and ilium was defined to be tied, which was also applied for the contact between the pins and the pelvis. The defined tie contact, constraining and loading conditions of the simulations were shown in Supplementary Figure S1.

We firstly performed a sensitivity analysis by increasing the element number in the simulation. Then, we considered two loading conditions, which shared a concentrated force of $500 \mathrm{~N}$ (a physiological loading of the body weight), [12, 13]. One condition occurred in the vertical direction, and the force was applied at the center of the top surface of the sacrum. The bottom of the pelvis was fixed by referring to a previous study where the acetabula were not taken into account neither [9]. The other was in the horizontal direction, and the force acted on the right peak point of the iliac crest

Table 2 Material parameters of the model

\begin{tabular}{llll}
\hline Items & Young's modulus (MPa) & Poisson's ratio & Element type \\
\hline Pelvis & & & \\
Cortical part & $12,000[10]$ & $0.3[10]$ & S3 \\
Spongy part & $100[10]$ & $0.2[10]$ & C3D4 \\
Sacrum & $12,000[10]^{a}$ & $0.3[10]^{a}$ & C3D4 \\
Fixator pin & $200,000[11]$ & $0.28[11]$ & C3D4
\end{tabular}

${ }^{a}$ Because the sacrum was treated to be only consisted of cortical bone, its materials parameters were set as the cortical part in pelvis while the left side of the iliac crest was fixed (see Supplementary Figure S1).

\section{Statistical analysis}

All data were presented as mean \pm standard deviation (SD) values. To evaluate whether the trajectories were precise, the parameters of external fixator pins including entry point, convergence angle and caudal angle were measured and compared before and after the operation by paired t-test. The $P$ value was two-sided, and values less than 0.05 were considered statistically significant. All statistical analyses were carried out using SPSS 13.0 (SPSS Inc., Chicago, IL, USA).

\section{Results}

Preoperative measurement and simulation operation

Using 3D reconstruction, the precise measurements of the pelvis were obtained. The entry point, direction of the pin, and the depth into the bone were successfully calculated using the software. Furthermore, the 3D printer accurately produced the pelvis model and navigation template using medical PLA (polylactic acid) material (Fig. 2a). The pin insertion channels in the template (Diameter: $6 \mathrm{~mm}$; Length: $30 \mathrm{~mm}$ ) were well matched to the external fixator pin (Diameter: $5 \mathrm{~mm}$; Length: 150 $\mathrm{mm}$ ). The insertion of pins was easily completed by the surgeon using the navigation templates. The evaluation of the pelvis model showed that the trajectories were consistent with the preoperative plans (Fig. 2b, c).

\section{Surgical outcome}

All external fixator pins were inserted using the individual drill template (Fig. 3). According to the postoperative CT scan, the average depth of all pins was $84.82 \pm 10.48$ $\mathrm{mm}$. No pin penetrated the cortex of the ilium during surgery (Fig. 4). The postoperative CT scan of the pelvis was obtained using similar scan parameters. Original DICOM data were imported into Mimics 17.0 software. The trajectories were precise and consistent with the preoperative plans (Fig. 5). No significant difference in the entry point, convergence angle, and caudal angle of the pins were noted before and after the operation (all $P>0.05$ ) (Table 3).

All eight patients were followed up for 3 months. No significant external fixation related complications occurred during the entire follow-up period, including loosening and rupture of pins, injury to blood vessels and nervous tissue, infection, and skin irritation. All patients were satisfied with the course and result of the treatment.

\section{The finite element model and mechanical analysis}

According to the sensitivity analysis by increasing the element number (from 204,071 to 320,081) in the 30 


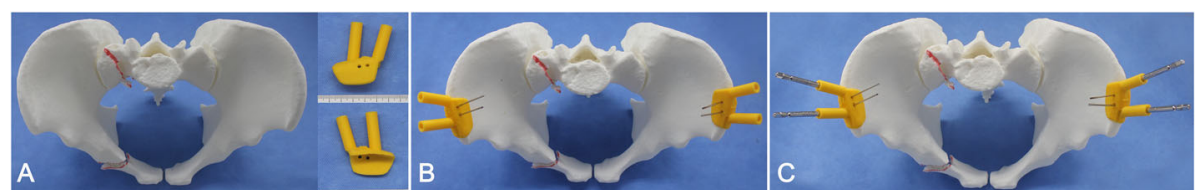

Fig. 2 Simulated operation with the 3D-printed model and navigation template. a The pelvis model and the navigation template. $\mathbf{b}$ The navigation template was matched to verify the degree of surface feature matching. The Kirschner wire needle was placed to fix the template. $\mathbf{c}$ The external fixator pins were inserted according to the navigation hole

mm model, we found a slight difference (less than $5 \%$ ) in the maximum von Mises stress of the pelvis, which guaranteed the model accuracy.

The von Mises stresses of the cortical part, spongy part and pins under vertical loading showed the roughly symmetrical distribution in both cases (Fig. 6). For the $30 \mathrm{~mm}$ case (Fig. 6a), the maximum stresses of the cortical and spongy parts were at the right-side entry point of the pelvis, they were $17.950 \mathrm{MPa}$ and $0.898 \mathrm{MPa}$, respectively. For the $70 \mathrm{~mm}$ case (Fig. 6b), the maximum stresses of the cortical and spongy parts were at the bottom $(7.097 \mathrm{MPa})$ and the leftside entry point $(1.895 \mathrm{MPa})$ of the pelvis. The maximum stress of pins in the $70 \mathrm{~mm}$ case was
68.220 MPa, which was greater than that $44.560 \mathrm{MPa}$ of the $30 \mathrm{~mm}$ case.

The von Mises stresses of the cortical part, spongy part and pins under horizontal loading resulted in an asymmetrical distribution (Fig. 7). For the $30 \mathrm{~mm}$ case (Fig. 7a), the maximum stresses of the cortical and spongy parts were at the right iliac fossa (19.690 MPa) and right-side entry point $(1.037 \mathrm{MPa})$ of the pelvis. For the $70 \mathrm{~mm}$ case (Fig. $7 \mathrm{~b}$ ), the maximum stresses of the cortical and spongy parts were at the left iliac fossa $(12.420 \mathrm{MPa})$ and right-side entry point $(1.099 \mathrm{MPa})$ of the pelvis. Different from the vertical loading, the maximum stress of pins in the $70 \mathrm{~mm}$ case was $71.840 \mathrm{MPa}$, which was less than that $93.710 \mathrm{MPa}$ of the $30 \mathrm{~mm}$ case.
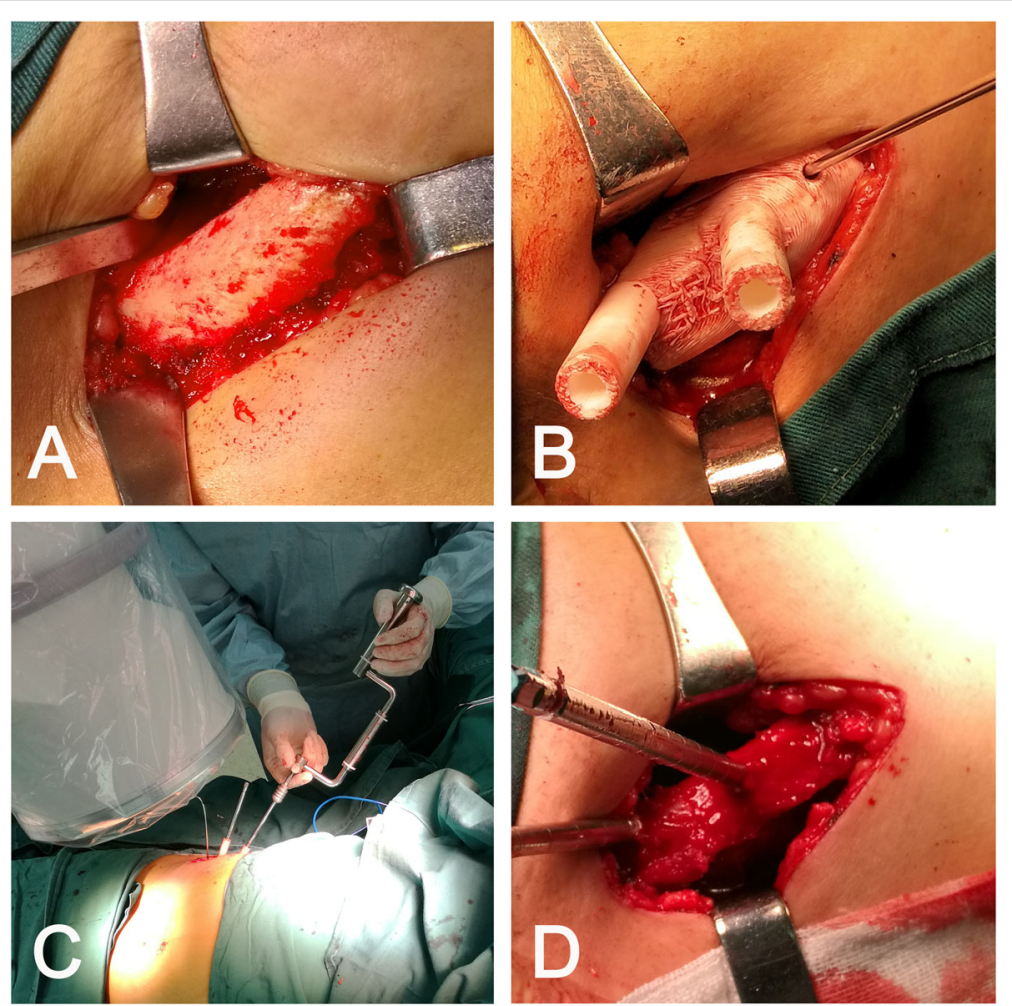

Fig. 3 The navigation template applied in the operation on a pelvis fracture patient. a The soft tissue was carefully separated to reveal the base of the iliac crest and the inner plate of the ilium. $\mathbf{b}$ The navigation template was matched to the iliac crest. $\mathbf{c}$ The navigation template was used to guide the insertion of the pin. $\mathbf{d}$ The intraoperative process was completed as performed in the simulated operation 


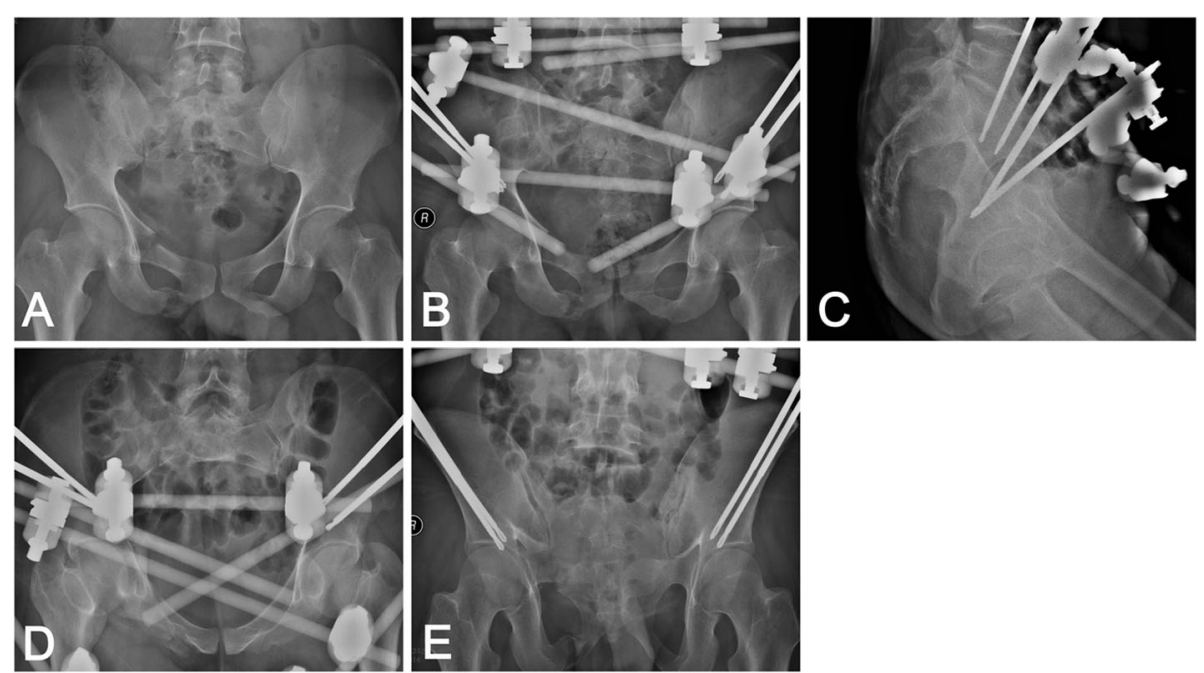

Fig. 4 Preoperative and postoperative $X$-ray of the pelvis. a Preoperative X-ray, $\mathbf{b}$ Postoperative $X$-ray in the AP plane, $\mathbf{c}$ Postoperative X-ray in the lateral projection, $\mathbf{d}$ Postoperative $\mathrm{X}$-ray in the inlet projection, e Postoperative $\mathrm{X}$-ray in the outlet projection

\section{Discussion}

In this study, all pins in simulation module was inserted from the iliac crest toward the supraacetabular region, and converged on the bone mass of the posterior column of the acetabulum. According to the postoperative $\mathrm{CT}$ scan, the pin insertion direction was well consistent with the preoperative plans. Furthermore, an individual navigation template was used as part of the surgical procedure for eight patients. Thirty-two external fixator pins were inserted using the individual navigation template. According to the postoperative CT scan, the average depth of the pins was $84.82 \mathrm{~mm}$. No pin penetrated the cortex of the ilium during the operation.

No significant difference in the entry point, convergence angle, and caudal angle of the pins were noted before and after the operation, indicating that the trajectories were precise and consistent with the preoperative plans. These findings were different from that of the previous studies, and our results showed that the pin position matched with that of the computer design [14, 15]. The match may be attributed to the following: First, due to the regular and superficial surface of the iliac crest, it was easy for the navigation template to fit the surface of the iliac crest well. Second, the thin layer CT scan reduced the distortion of data conversion and 3D construction, which effectively controlled the error in data transformation and editing. Third, the design of the pin insertion channel was based on digital 3D construction, which could find a suitable path from multiple planes, and improve accuracy and repeatability during the operation. Fourth, during template designing, the identification mark in multiple planes improved the ability to fit the surface of the iliac crest, and long insertion channels in the template controlled the pin insertion position effectively. Finally, medical PLA material with certain hardness and toughness was selected for the template preparation, which proved to be convenient for preoperative disinfection and intraoperative application.

The primary treatment modality in case of a pelvic fracture focuses on controlling bleeding, restoring stability, and preventing complications. The instability of the pelvis fracture is the major reason for excessive bleeding. Bleeding is often difficult to control; therefore, the reduction and fixation need to be performed as soon as a pelvis fracture is diagnosed [16]. Balbachevsky et al. reported that external fixation is used for more than $79.5 \%$ of patients with a pelvic fracture, especially for patients with multiple injuries and soft tissue damage (1). However, clinical follow-up results showed that external fixation frequently causes infections and pin loosening (in more than 50\% patients) and does not effectively stabilize the pelvis, indicating that the current method of external fixation needs to be improved clinically $[17,18]$.

In a previous study by Hiesterman et al., either a locking reconstruction plate or a spinal rod was placed through a subcutaneous tunnel, and the fixation into the iliac crest and pubis was achieved to effect stability. At 6-month follow-up, there was only one pin tract infection out of all the 11 patients [19]. Noda et al. reported that the biplanar external fixation could provide better stability than the uniplanar external fixation and minimize pin-site infection [20]. Archdeacon et al. compared the structural stiffness of a pin construct with 2 models of parallel pin constructs and found a significantly stiffer construct for in-plane loading compared 

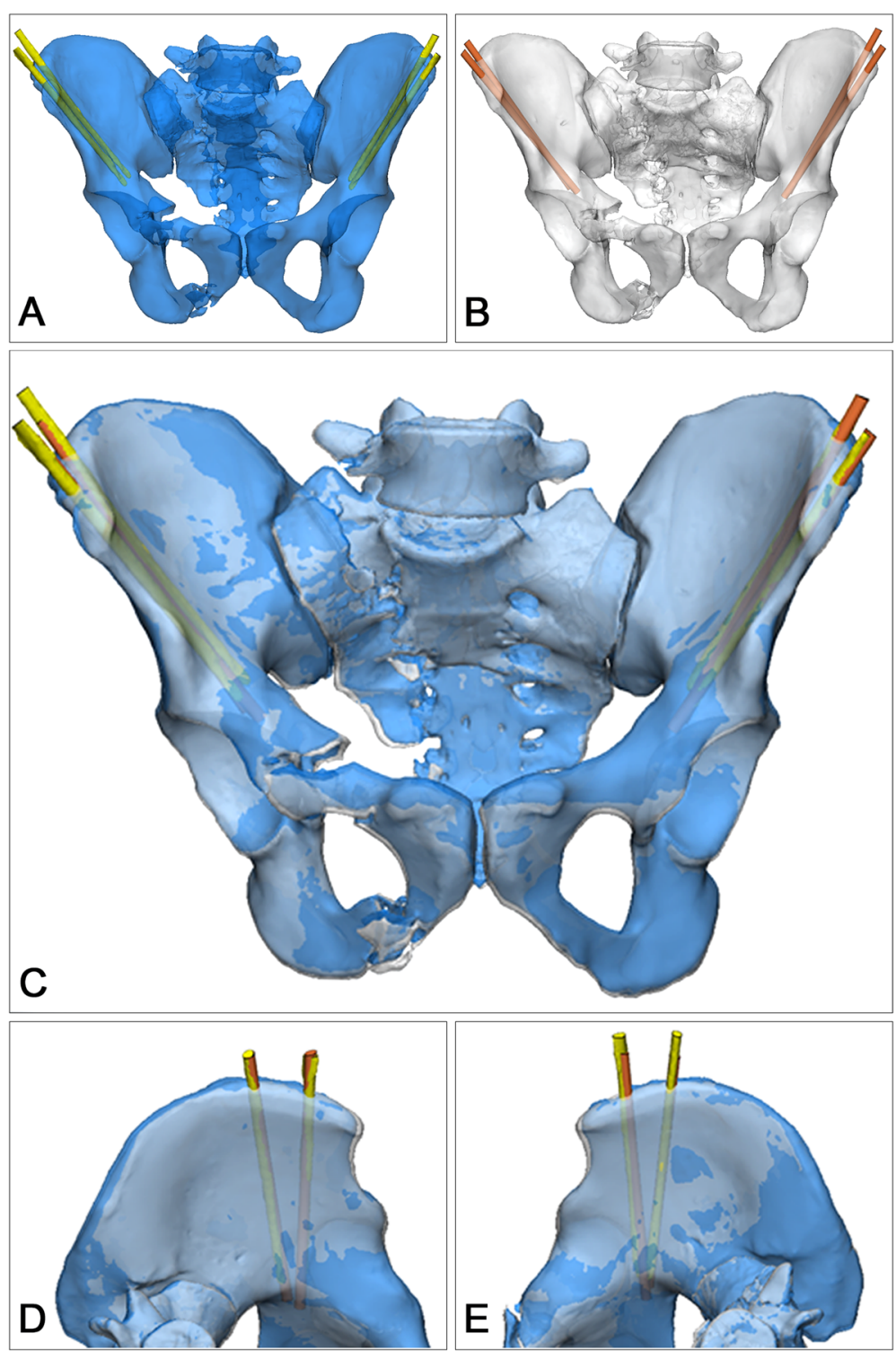

Fig. 5 The overlay of trajectories before and after the operation. The trajectories after the operation (a) showed all pin was inserted pointing to the posterior column of the acetabulum, consistent with those in the preoperative plans $(\mathbf{b})$. The merged images (c-e) confirmed the preoperative and postoperative trajectories were mostly overlaid by visual inspection

with either parallel pin construct [21]. Meanwhile, a few reports have supported that external fixation on supraacetabulum has a good stiff construct because the bone condition of supra-acetabulum is beneficial for the deep pin position. Therefore, there are several limitations of

Table 3 Comparison of parameters before and after the surgery

\begin{tabular}{llll}
\hline Parameters & Before surgery & After surgery & $p$ \\
\hline$d(\mathrm{~mm})$ & $41.07 \pm 11.07$ & $41.16 \pm 10.97$ & 0.883 \\
$\mathrm{~A}\left({ }^{\circ}\right)$ & $40.59 \pm 7.83$ & $40.89 \pm 7.93$ & 0.555 \\
$\mathrm{~B}\left(^{(}\right)$ & $36.10 \pm 8.69$ & $36.62 \pm 9.12$ & 0.510 \\
\hline
\end{tabular}

$d$ : The distance from the entry point to the anterior superior iliac spine. A: The convergence angle. B: The caudal angle external fixation on supra-acetabulum. First, it is difficult to expose the anterior inferior iliac spine, identify the entry point, and control pin insertion position [22]. Second, the pin insertion requires splitting of the sartorius and iliac muscles, which may cause an injury to the lateral femoral cutaneous nerve and irritates the deep soft tissue [23]. Third, although the tip of the pin in the supra-acetabulum could reach the sacroiliac joint, the average depth of the pin position using the standard method was approximately $50 \mathrm{~mm}$ [6], and the insertion channel is surrounded by cancellous bone resulting in a broad fixed area, which influences the mechanical properties of the external fixation. 


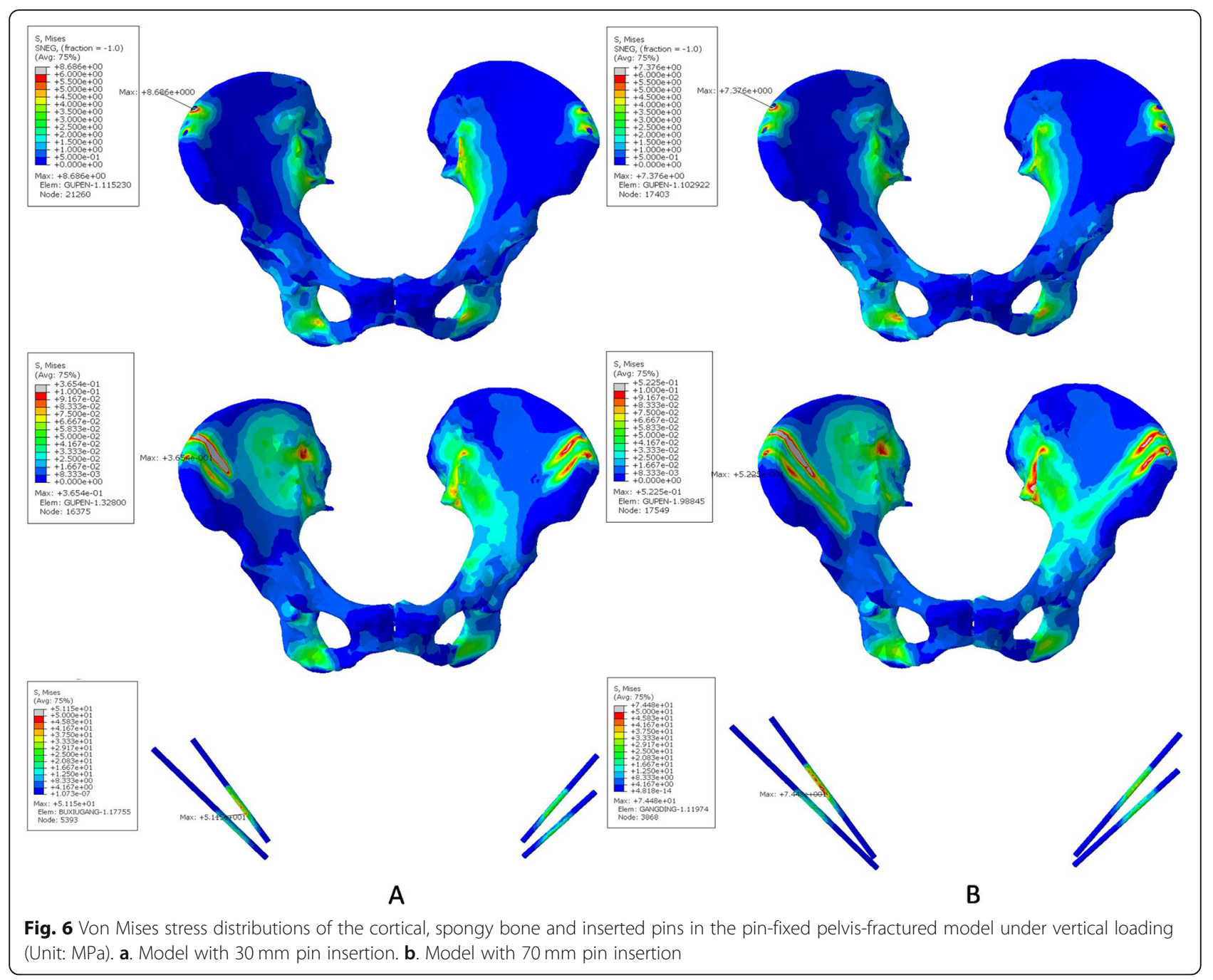

The stability and pin-related complications following external fixation is closely related to the pin insertion position $[6,7,24]$. Gardner et al. reported that the external fixation could achieve compression and stabilization of the sacroiliac joint when the pins were placed completely to the posterior superior iliac spine [6]. Ponsen et al. reported that external fixator of pelvic ring could improve stability for the treatment of type $\mathrm{C}$ pelvic fracture by an increase in pin diameter and alternative pin position [7]. The high rate of infection and pin-related loosening was caused by the poor direction and shallow depth of the external fixator pin using the traditional external fixation on the iliac crest [24]. In the present study, the pin insertion procedure was modified from that of the traditional method. All pins were inserted into the supraacetabular region, where the highest bone density in the human body could be found [6]. The individual template could improve the precision of the external fixator pin position, and increase the depth compared with the traditional method $(50 \mathrm{~mm})$ [4]. No pin-related complication such as infection and loosening were found during the postoperative follow-up. Finite element analysis showed the deeper pin position $(70 \mathrm{~mm}$ in our study) reduced the maximum stress of the cortical part but slightly increased the counterpart of the spongy part. This indicated that the deeper pin position could rationalize the maximum von Mises stresses of the pelvis.

There are several limitations to our current study. First, compared with percutaneous approach in the traditional method, our method requires stripping of the soft tissue on the iliac crest and the inner plate of the ilium for the template attaching, which cause about $4 \mathrm{~cm}$ skin incision and local soft tissue invasions. Moreover, it is noted that such method may not be suitable for emergency surgeries as it will take about $4 \mathrm{~h}$ for the preparation and $2 \mathrm{~h}$ for sterilization of individual templates before operation. Second, the 


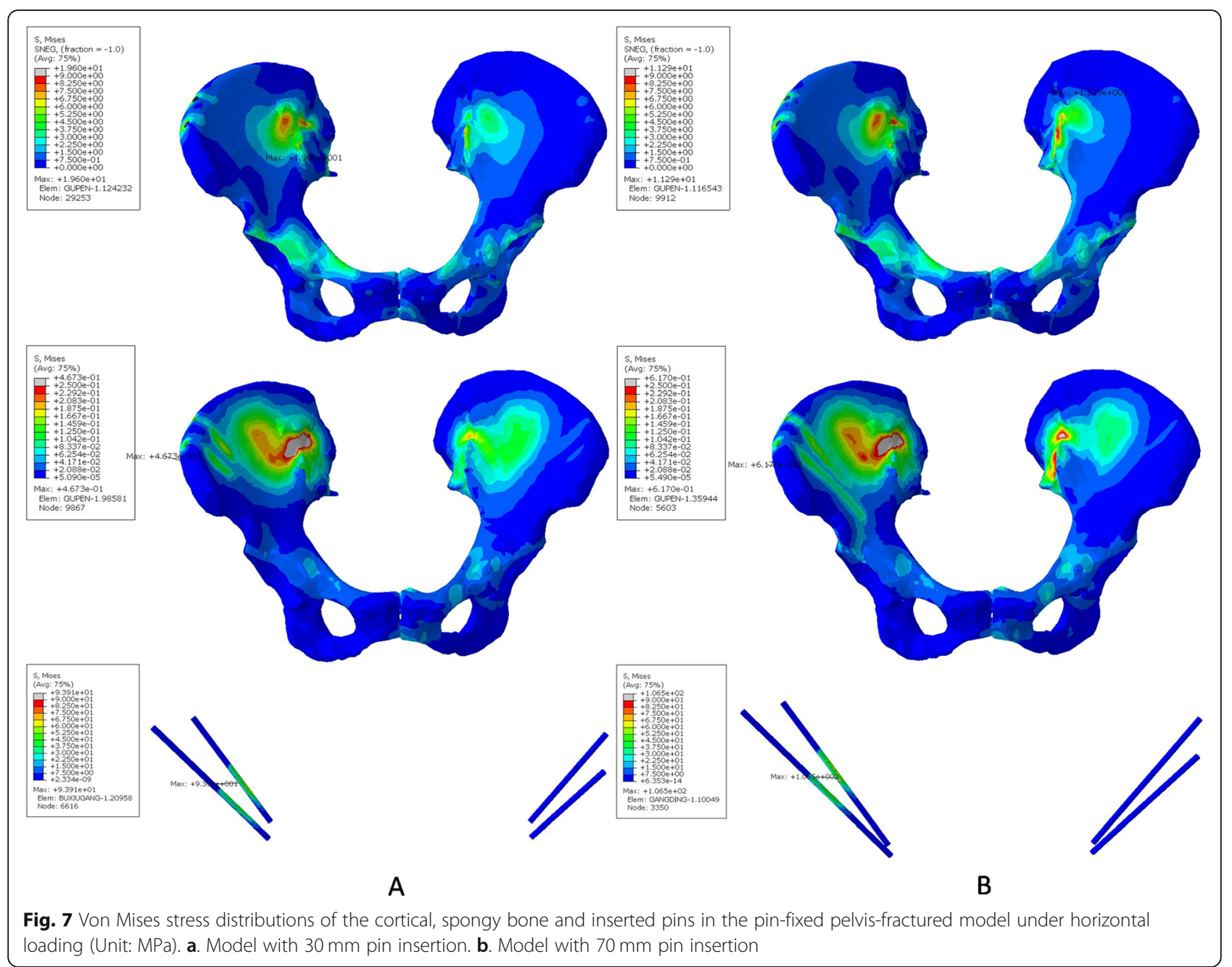

number of patients was relatively small, more patients are warranted to confirm the reproducibility of our study. The mechanical difference among several external fixation methods should be analyzed to strengthen the statistic power in the future study. Moreover, strictly validating the model in the current study was difficult because cadaveric specimens from available donors was absent. A high credibility of the model should be provided by in vitro experiments with cadavers for potential clinical use in the future [25].

\section{Conclusions}

The individual navigation template is an improvement of the traditional method. The method could increase precision and depth of pin position. The deeper pin position could rationalize the maximum von Mises stresses of the cortical and spongy bones of the pelvis. Finally, the approach resulted in good mechanical stability and low risk of pin-related complications.

\section{Supplementary information}

Supplementary information accompanies this paper at https://doi.org/10. 1186/s12891-020-03509-6.

Additional file 1: Figure S1. The defined tie contact, constraining and loading conditions in the simulations. (A) Definition of the tie contact was shown in the simulations. (B) One condition occurred in the vertical direction, and the force (i.e., RP-1) was applied at the center of the top surface of the sacrum. To be consistent with the surgical procedure, points $\mathrm{a}, \mathrm{b}, \mathrm{c}, \mathrm{d}$ were defined as the locations of the connectors, and the distances between points $a, b, c, d$ and entry points of the pelvis were uniformly set as $30 \mathrm{~mm}$. The four points were coupled with the defined four reference pins (RP3-RP6) on which the constraining conditions were applied. (C) The other condition was in the horizontal direction, and the force (i.e., RP-2) acted on the right peak point of the iliac crest while the left side of the iliac crest was fixed.

Abbreviations

RP: Rapid prototyping; PLA: polylactic acid

Acknowledgements None. 


\section{Authors' contributions}

BL performed the experiments, participated in collecting and analyzing the data, and drafted the manuscript. QC and SC made the finite element analysis, and QC drafted the methods, results and discussion about finite element analysis. SL and QY performed the experiments. BW and YX collected the data. CT and LW designed the study, participated in analyzing the data, and helped to draft the manuscript. All authors read and approved the final manuscript.

\section{Authors' information}

Not applicable.

\section{Funding}

This work was financially supported by the National Nature Science Foundation of China (No. 81702205, 81702148, 81771985) and Nature Science Foundation of Jiangsu Province (No. BK20170141, BK20170139).

\section{Availability of data and materials}

The datasets analyzed during the current study are available from the corresponding author on reasonable request.

\section{Ethics approval and consent to participate}

This study was approved by the Ethics Committee of Nanjing First Hospital (Permit Number KY20170401-04). We obtained written informed consent from all the participants before publishing this information.

\section{Consent for publication}

Not applicable.

\section{Competing interests}

The authors declare that they have no competing interests.

\section{Author details}

'Department of Orthopaedic Surgery, Nanjing First Hospital, Nanjing Medical University, Nanjing 210006, China. ${ }^{2}$ School of Biological Science \& Medical Engineering, Southeast University, Nanjing, China. ${ }^{3}$ Digital Medicine Institute, Nanjing Medical University, Nanjing 210006, China. ${ }^{4}$ Department of Orthopaedic Surgery, Xuzhou Central Hospital, Xuzhou 221000, China. ${ }^{5}$ Engineering Research Center in Biomaterials, Sichuan University, Chengdu 610065, China

Received: 20 October 2019 Accepted: 15 July 2020

Published online: 21 July 2020

\section{References}

1. Saydam M, Şahin M, Yılmaz KB, Tamam S, Demir P, Akıncı M. Correlation of pelvic fractures and associated injuries: an analysis of 471 pelvic trauma patients. Ulus Travma Acil Cerrahi Derg. 2019;25(5):489-96.

2. Brown JV, Yuan S. Traumatic injuries of the pelvis. Emerg Med Clinics. 2020; 38(1):125-42.

3. Balbachevsky D, Belloti JC, Doca DG, Jannarelli B, Junior JAY, Fernandes HJA, et al. Treatment of pelvic fractures-a national survey. Injury. 2014;45:S46-51.

4. Waikakul S, Harnrungroj T, Vanadurongwan V. Immediate stabilization by external fixator in unstable fractures of the pelvis: results in 214 patients. Thai J Surg. 1997;18(2):59-65.

5. Wright RD Jr. Indications for open reduction internal fixation of anterior pelvic ring disruptions. J Orthop Trauma. 2018;32:S18-23.

6. Gardner MJ, Kendoff D, Ostermeier S, Citak M, Hüfner T, Krettek C, et al. Sacroiliac joint compression using an anterior pelvic compressor: a mechanical study in synthetic bone. J Orthop Trauma. 2007;21(7):435-41.

7. Solomon L, Pohl A, Chehade M, Malcolm A, Howie D, Henneberg M. Surgical anatomy for pelvic external fixation. Clin Anat. 2008;21(7):674-82.

8. Kj P, Joosse P, Hoek Van Dijke GA, Snijders CJ. External fixation of the pelvic ring: an experimental study on the role of pin diameter, pin position, and parasymphyseal fixator pins. Acta Orthop. 2007;78(5):648-53.

9. El-Rich M, Villemure I, Labelle H, Aubin C. Mechanical loading effects on isthmic spondylolytic lumbar segment: finite element modelling using a personalised geometry. Comp Methods Biomech Biomed Eng. 2009;12(1): 13-23.

10. Grauer JN, Biyani A, Faizan A, Kiapour A, Sairyo K, Ivanov A, et al. Biomechanics of two-level Charite artificial disc placement in comparison to fusion plus single-level disc placement combination. Spine J. 2006:6(6):659-66.

11. Material Properties Database. 2020. https://www.makeitfrom.com. Accessed 28 Mar 2020.

12. Osterhoff G, Dodd AE, Unno F, Wong A, Amiri S, Lefaivre KA, et al. Cement augmentation in sacroiliac screw fixation offers modest mechanical advantages in a cadaver model. Clin Orthop Relat Res. 2016;474(11):2522-30.

13. Arkusz K, Klekiel T, Niezgoda TM, Będziński R. The influence of osteoporotic bone structures of the pelvic-hip complex on stress distribution under impact load. Acta of Bioengineering Biomech. 2018;20(1):29-38.

14. Kaneyama S, Sugawara T, Sumi M. Safe and accurate midcervical pedicle screw insertion procedure with the patient-specific screw guide template system. Spine. 2015;40(6):E341-8.

15. Yang F, Yao S, Chen K-f, Zhu F-z, Xiong Z-k, Ji Y-h, et al. A novel patient-specific three-dimensional-printed external template to guide iliosacral screw insertion: a retrospective study. BMC Musculoskelet Disord. 2018;19(1):397.

16. Riemer BL, Butterfield SL, Diamond DL, Young JC, Raves JJ, Cottington E, et al. Acute mortality associated with injuries to the pelvic ring: the role of early patient mobilization and external fixation. J Trauma. 1993;35(5):671-5 discussion 6-7.

17. Mason W, Khan S, James C, Chesser T, Ward A. Complications of temporary and definitive external fixation of pelvic ring injuries. Injury. 2005;36(5):599-604.

18. Hua X, Yan SG, Cui Y, Yin Z, Schreiner AJ, Schmidutz F. Minimally invasive internal fixator for unstable pelvic ring injuries with a pedicle screw-rod system: a retrospective study of 23 patients after 13.5 months. Arch Orthop Trauma Surg. 2019;139(4):489-96.

19. Hiesterman TG, Hill BW, Cole PA. Surgical technique: a percutaneous method of subcutaneous fixation for the anterior pelvic ring: the pelvic bridge. Clin Orthop Relat Res. 2012;470(8):2116-23.

20. Noda M, Yamakawa T, Fujii T. The use of a Biplanar construct in pelvic external fixation. Orthopedics. 2009;32(6):448.

21. Archdeacon MT, Arebi S, Le TT, Wirth R, Kebel R, Thakore M. Orthogonal pin construct versus parallel uniplanar pin constructs for pelvic external fixation: a mechanical assessment of stiffness and strength. J Orthop Trauma. 2009; 23(2): 100-5

22. Chana-Rodríguez F, Cuervas-Mons M, Rojo-Manaute J, Mora F, Arnal J, Vaquero-Martín J. Ultrasound-guided supra-acetabular pin placement in pelvic external fixation: description of a surgical technique and results. Injury. 2017:48:S66-74

23. Haidukewych GJ, Kumar S, Prpa B. Placement of half-pins for supraacetabular external fixation: an anatomic study. Clin Orthop Relat Res. 2003; 411:269-73

24. Liu J, Lai K-A, Chou Y-L. Strength of the pin-bone interface of external fixation pins in the iliac crest. A mechanical study. Clin Orthopaedics Related Res. 1995:310:237-44.

25. Viceconti M, Olsen S, Nolte L-P, Burton K. Extracting clinically relevant data from finite element simulations. Clin Biomech. 2005;20(5):451-4.

\section{Publisher's Note}

Springer Nature remains neutral with regard to jurisdictional claims in published maps and institutional affiliations.

Ready to submit your research? Choose BMC and benefit from:

- fast, convenient online submission

- thorough peer review by experienced researchers in your field

- rapid publication on acceptance

- support for research data, including large and complex data types

- gold Open Access which fosters wider collaboration and increased citations

- maximum visibility for your research: over $100 \mathrm{M}$ website views per year

At BMC, research is always in progress.

Learn more biomedcentral.com/submissions 